\section{Notes on coronectomy}

\author{
T. Renton ${ }^{1}$
}

VERIFIABLE CPD PAPER

IN BRIEF
Outlines the indications for coronectomy.
- Outlines the potential consequences of
coronectomy.
Increases awareness of the coronectomy
technique among general dental
practitioners.

IN BRIEF

lncreases aware

echnique among general dental

practitioners.

Coronectomy involves removal of a crown from roots of a healthy tooth in healthy patients indicated to prevent inferior alveolar nerve injury in a high risk case. Since the original paper in 2005 (Br J Oral Maxillofac Surg 2005; 43: 7-12) describing a prospective randomised study on coronectomy, there has been a lot of interest in this procedure and it has become routine practice in many oral surgery departments within the UK and USA. A significant variance in thresholds for prescribing coronectomy and also for the technique of its delivery has been highlighted by a recent review. This has resulted in frequent queries about the technique and which patients may be suitable. Thus this paper aims to highlight some finer points of the coronectomy technique and how to avoid potential pitfalls.

\section{INTRODUCTION}

A recent review of coronectomy procedures $^{1}$ has brought to light the significant variance in thresholds for not only prescribing coronectomy, but the technique of delivering the procedure. Third molar surgery related to inferior alveolar nerve injury is reported to occur in up to $3.6 \%$ cases permanently and $8 \%$ cases temporarily., ${ }^{2,3}$ Factors associated with inferior alveolar nerve injury (IANI) are age, difficulty of surgery and proximity to the IAN canal. If the tooth is closely associated with the IAN canal radiographically, 20\% of patients having these teeth removed are at risk of developing temporary IAN nerve injury and $1-4 \%$ are at risk of permanent injury. ${ }^{2-6}$

Radiographic signs indicative of possible IAN risk include:

- Diversion of the canal

- Darkening of the root

- Narrowing of the root/canal

- Interruption of the canal lamina dura

- Interruption of the juxta-apical area.

If these plain film radiographic risk factors are identified (Figs 1a and 1b), removal

Professor, Oral Surgery, Kings Health Partners Dental Institute, Bessemer Road, London, SE5 9RS

Correspondence to: Tara Renton

Email: tara.renton@kcl.ac.uk; Tel: 02032992313

\section{Refereed Paper}

Accepted 3 March 2011

DOI: 10.1038/sj.bdj.2012.265

${ }^{\circledR}$ British Dental Journal 2012; 212: 323-326

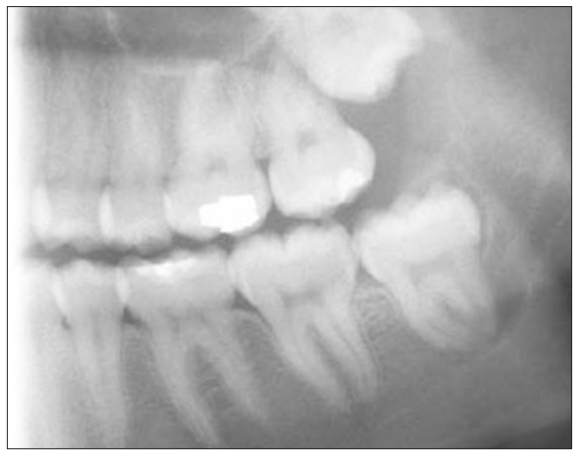

Fig. 1a DPT High risk left M3M with juxta apical area and darkend roots

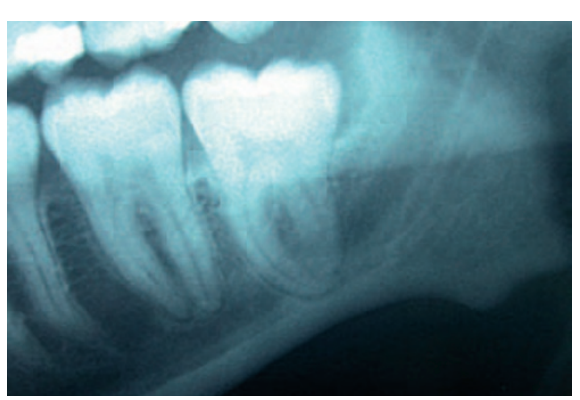

Fig. 1b DPT high risk left M3M with darkening of roots

of the third molar will result in elevated risk of IANI ( $2 \%$ permanent and 20\% temporary)..$^{3-6}$ If the tooth is 'high risk', carious and/or the patient is medically compromised, the tooth must be extracted and the patient must be informed about the elevated risk of nerve injury.

Coronectomy reduces the likelihood of nerve injury by ensuring retention of the vital roots when they are close to the

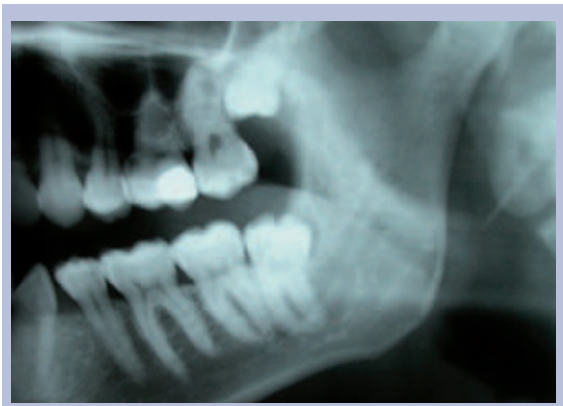

Fig. 2a DPT preoperative coronectomy left M3M

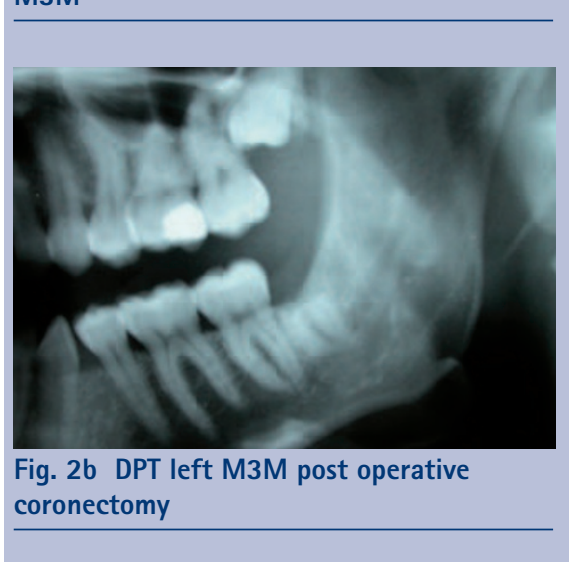

inferior alveolar canal (as estimated on radiographs or CBCT). The method aims to remove only the crown (all enamel) of an impacted mandibular third molar while leaving the root and pulp undisturbed, thereby avoiding direct or indirect damage to the IAN (Figs 2a and 2b).

The six latest articles on coronectomy consist of two randomised controlled trials, two prospective cohort studies, one case 
control study and one retrospective study. ${ }^{6-13}$ Three of the six articles investigate the fate of third molars deemed at high risk on a DPT radiograph that have been coronected. ${ }^{7-9}$ The remaining three articles compare the techniques of coronectomy versus complete removal of the high risk third molar tooth. ${ }^{6,10,11}$ Interestingly, Dolanmaz et al. ${ }^{11}$ did not have a high risk control group for comparison as they deemed this unethical. All six articles suggested that the technique of coronectomy had merit and many practitioners regularly use the coronectomy approach in order to minimise IANIs.

\section{TECHNIQUE}

\section{Preoperative assessment}

\section{The patient}

Coronectomy is an alternative procedure to complete extraction when a tooth is deemed 'high risk' (crossing both lamina dura of the ID canal on a plain film) but vital and in a patient who is not medically compromised (not immune compromised [diabetic, long term steroids, chemotherapy, HIV]; or potential poor healing [previous irradiation]). Patients should be fully cognisant of the potential risks of a 'dry socket' (incidence 5\%) and the additional possibility of a second surgical procedure either early or late postoperatively.

\section{Radiography}

The mandibular third molar is identified as high risk based on routine plain film radiography (Figs 1a and 1b). Once identified as a high risk, a cone beam CT is essential in confirming the relationship of the tooth to the inferior alveolar canal (Figs 3a-d).

A recent study has explicitly highlighted the link between the plain radiographic features and cone beam CT findings. ${ }^{15}$ Umar et al. reviewed 50 cases that had been identified as high risk on plain films and then correlated the CBCT findings with their appearance. Loss of lamina dura of the canal was linked to contact with the canal in all cases, darkening was related to canal cortical loss in $78 \%$ of cases, contact with the nerve in 60\% and grooving of the root in one case. This study may reinforce the possibility that plain film assessment and planned coronectomy may negate the need for additional CBCT scanning and its related irradiation. However, as the tooth roots may be mobilised during the coronectomy procedure it may be argued that the surgeon should be fully cognisant of the relationship between the tooth roots and IAN canal to ensure minimal morbidity of the nerve should the roots be mobilised and require removal. In addition, if CBCT confirms that the mandibular third molar roots are distinct from the ID canal (Figs 3a and 3b) or inextricably involved with the IAN canal (Figs 3c and 3d) the surgeon may elect to ensure mobilisation of the roots is prevented by extended sectioning of the crown from the roots, but this technique may be associated with increased risk to the lingual nerve. ${ }^{9}$

CBCT has provided an additional indication for elective coronectomy, which is when there is loss of the lingual cortex (identified in 30\% of cases) and IDC buccally with a mandibular third molar root apex 'sandwiched' in between (Fig. 4). This minimises injury to the IDC (IAN) during attempted elevation of the root, with a high risk of loss of the tooth fragment into the submandibular space.

Also if the patient or tooth is compromised the CBCT scan will enable appropriate planning for removal of the high risk mandibular third molar with an appropriate consent.

CBCT does involve the additional radiation dose of 60-120 $\mu \mathrm{Sv}$ which must not be forgotten but is appropriate in planning to minimise IAN injury in relation to mandibular third molar surgery.

The routine use of CBCT with its attendant high radiation dose is justified in that, based on CBCT findings, only 5-15\% of the high risk $\mathrm{M} 3 \mathrm{M}$ cases will proceed to coronectomy, depending on the surgeon involved. Thus significantly fewer patients will have coronectomied retained roots, for protection of their inferior alveolar nerve, without the additional risk of future infection and resultant necessary additional surgery for root removal.

\section{Consent}

Taking into account the above, the patient therefore must be consented with the words 'it is intended for a coronectomy to be undertaken, however, if the roots are mobilised during surgery they will require removal with the heightened risk of nerve injury'. ${ }^{6}$ The patient must also be aware that there is a risk of early and late infection that will necessitate the removal of the roots, which in most cases occurs once

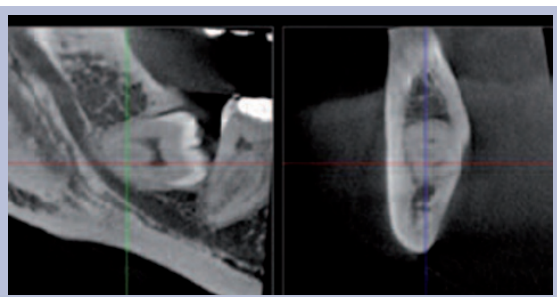

Fig. 3a CBCT right M3M distant with ID canal inferior and corticated. Remove M3M

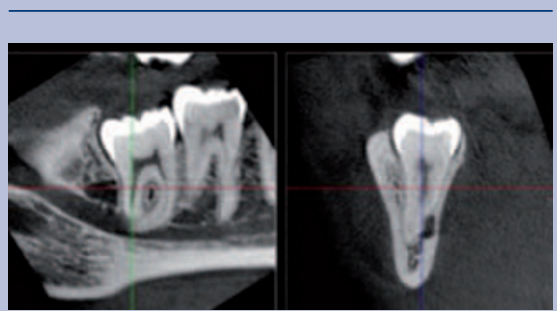

Fig. 3b CBCT nerve proximal and lingual with decortication of ID canal. Remove M3M

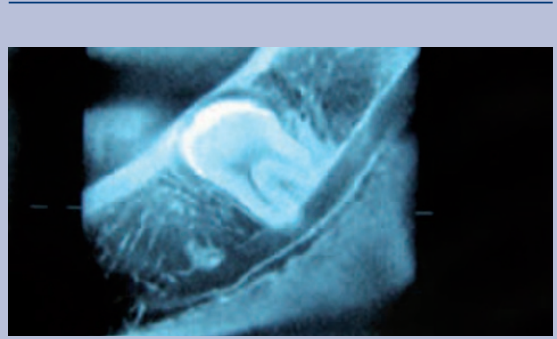

Fig. 3c CBCT left M3M roots straddling ID canal

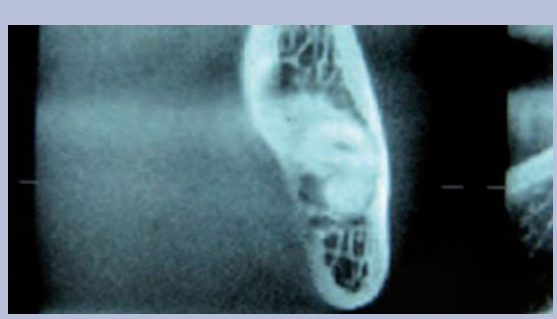

Fig. 3d CBCT of bifid ID nerve winding between roots of left M3M. Coronectomy

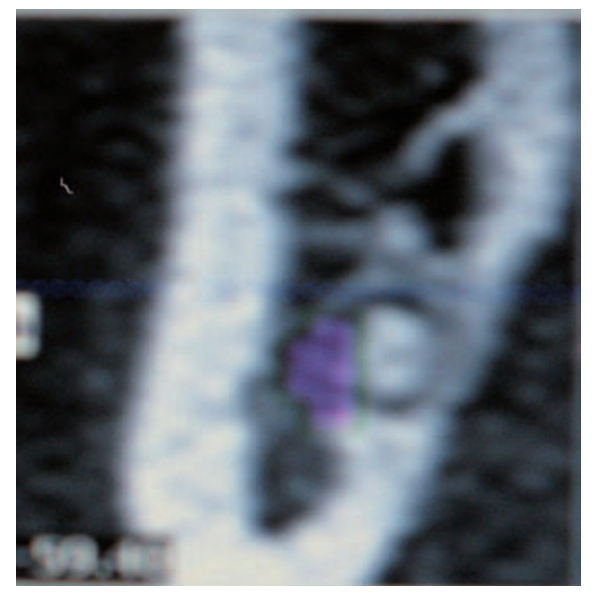

Fig. 4 CBCT close up left M3M root sandwiched between ID nerve (purple) and loss of lingual plate

the tooth root has erupted away from the IAN canal but in some cases when the root is perforated by the nerve, dragging the nerve superiorly as it erupts. ${ }^{14}$ 


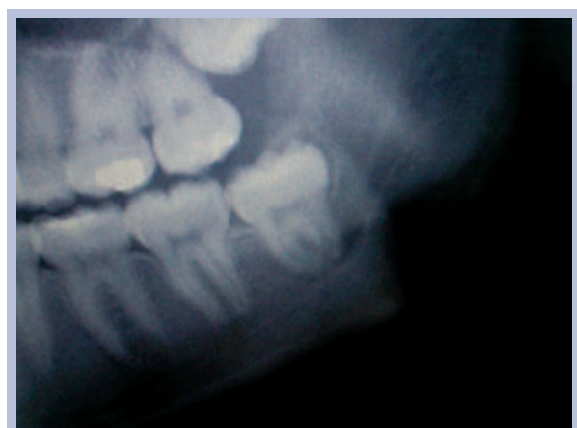

Fig. 5a Coronectomy preop left DPT high risk $\mathrm{M} 3 \mathrm{M}$

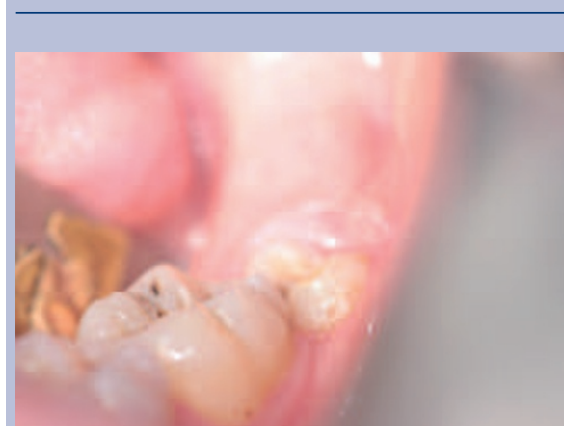

Fig. 5b Coronectomy preop partially erupted M3M

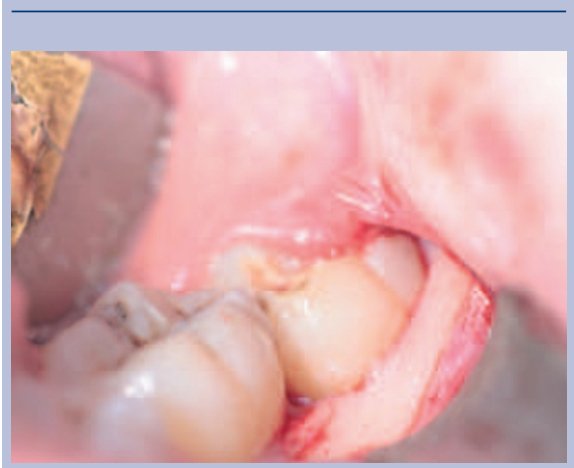

Fig. 5c Coronectomy elevated small buccal triangular flap with bone exposure

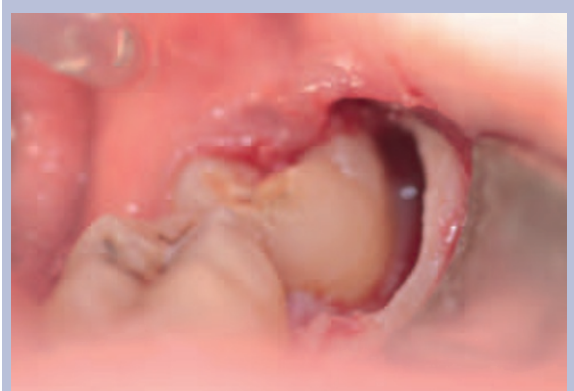

Fig. $5 d$ Coronectomy with buccal bone removal using fissure bur

\section{The operative technique (a coronectomy case as illustrated in Fig. 5)}

1. Long buccal infiltration and anterior buccal infiltration (4 $\mathrm{ml}$ articaine 4\%) is given as with routine lower third molar surgery. An inferior dental

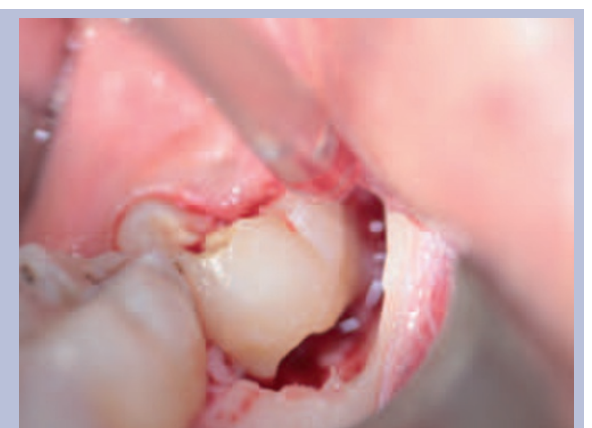

Fig. 5e Coronectomy with section into pulp chamber for decoronation of crown

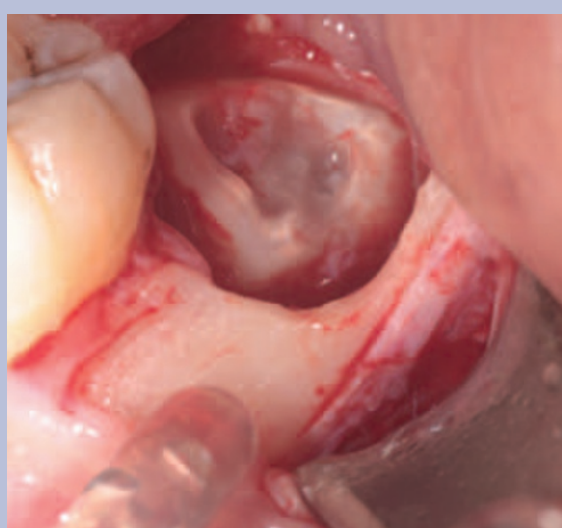

Fig. $5 f$ Coronectomy illustrating cut surface of retained roots with pulpal exposure

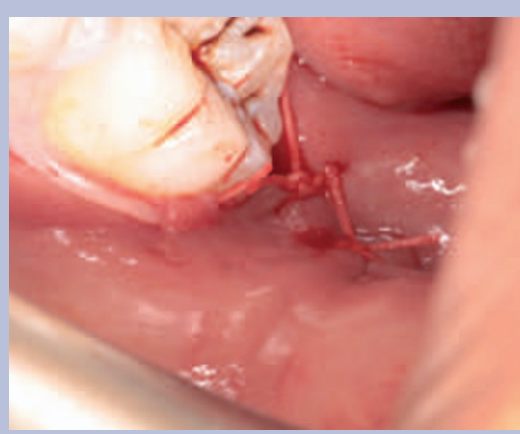

Fig. $5 g$ Coronectomy after wound toilet and irrigation the buccal triangular flap is replaced with 40 vicryl rapide suture

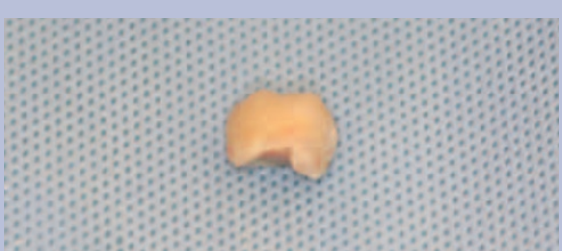

Fig. 5h Coronectomy crown fragment, always inspect root surface to ensure all enamel is removed

block (lidocaine 2\%) may be used in addition if necessary

2. A buccal triangular mucoperiosteal full thickness flap is raised to expose the third molar tooth (no lingual access)

3. A fissure bur is used to create a buccal gutter of bone adjacent and buccal to the tooth (not distal) and to expose the amelo-cemental junction (ACJ). This would be similar access as that gained for application points for third molar elevation but in this scenario we are trying to gain access to cut through the ACJ into the pulp

4. A fissure bur is then used and drilled directly into the pulp at the buccal groove intersection with the ACJ. This cut is then lateralised to create a narrow horizontal groove in the tooth just below the ACJ. The depth of this cut needs to be into the pulp then lateralised but no more than the length of the fissure bur so has to avoid perforation of the lingual cortical plate, the distal and mesial confines of the tooth (in other studies the whole crown is sectioned from the roots, eg Pogrel et $a l)^{9}$

5. A small elevator instrument such as a Coupland No. 1 or a straight Warwick James is used to fracture off the crown from the roots. Care must be taken not to apply too much torque to the tooth at this point, so the risk of root mobilisation is minimised. The crown of a mesioangularly or horizontally impacted third molar may need further sectioning to avoid damage to the lower second molar tooth

6 . If at any time the roots are mobilised they should be removed

7. A rose-head bur is then used, if necessary, to remove any enamel spurs and to take the level of the remaining root a few millimetres below the alveolar crestal bone level. The pulp chamber tissue should be left untouched and untreated during smoothing of the root surface in order to maintain vitality of the root. Ideally there should be alveolar bone above the root edges but this is not always possible where bone levels are absent (for example the missing lingual plate)

8. The area is then closed primarily with resorbable sutures, usually a single suture (4/0 vicryl)

9. The author prefers pre- and postoperative chlorhexidine mouth wash or gel. Antibiotics are not prescribed unless there is a concurrent pericoronal infection. 


\section{Postoperatively}

\section{Early}

Should the patient return with 'dry socket' symptoms, treat as one would a dry socket with irrigation using chlorhexidine solution and placement of a resorbable dressing (Alvogyl). If the patient returns with persistent or recurrent infection then consideration should be given to removing the roots. In the author's experience, two cases have had early postoperative infection with IAN neuritis (intermittent lip paraesthesia resolving with antibiotics) due to proximal apical infection to the IAN canal and in both cases this resolved with extraction of the infected roots.

Late

It is estimated that eruption of the "coronectomised' roots may occur in 2-5\% of cases at 2-5 years. Often the roots will have erupted away from the IAN canal thus minimising IAN morbidity, however, in some cases the root may be intricately involved with the nerve and careful surgery is required to separate the root from the nerve. ${ }^{14}$

Remember it is possible for any mandibular posterior tooth to be proximal to the IAN. Be aware that any mandibular tooth that is crossing the IAN canal and displays the radiographic signs is associated with increased risk of IAN injury. In these circumstances, the patient must be assessed, consented and treated similarly to high risk third molar teeth.

Coronectomy clincal photography provided by $\mathrm{Dr} G$ Umar, Associate Specialist in Oral Surgery, KHP.

1. Frafjord R, Renton T. A review of coronectomy. Oral Surg 2010; 3: 1-7.

2. Howe $G \mathrm{~L}$, Poyton $\mathrm{H}$ G. Prevention of damage to the inferior dental nerve during the extraction of mandibular third molars. Br Dent J 1960; 109: 355-363.

3. Rood J P, Shehab B A. The radiological prediction of inferior alveolar nerve injury during third molar surgery. Br J Oral Maxillofac Surg 1990; 28: 20-25.

4. Rud J. Third molar surgery perforation of the inferior dental nerve through the root. Tandlaegebladet 1983; 87: 659-667

5. Rud J. Third molar surgery: relationship of root to mandibular canal and injuries to inferior dental nerve. Tandlaegebladet 1983; 87: 619-631.

6. Renton T, Hankins M, Sproate C, McGurk M. A randomised controlled clinical trial to compare the incidence of injury to the inferior alveolar nerve as a result of coronectomy and removal of mandibular third molars. Br J Oral Maxillofac Surg 2005; 43: 7-12

7. Friedland B, Donoff B, Dodson T B. The use of 3-dimensional reconstructions to evaluate the anatomic relationship of the mandibular canal and impacted mandibular third molars. J Oral Maxillofac Surg 2008; 66: 1678-1685

8. O'Riordan B C. Coronectomy (intentional partial odontectomy of lower third molars). Oral Surg Oral Med Oral Path Oral Radiol Endod 2004; 98: 274-280.

9. Pogrel M A. Coronectomy: a technique to protect the inferior alveolar nerve. J Oral Maxillofac Surg 2004; 62: 1447-1452.

10. Hatano Y, Kurita K, Kuroiwa Y, Yuasa H, Ariji E. Clinical evaluations of coronectomy (intentional partial odontectomy) for mandibular third molars using dental computed tomography: a case-control study. J Oral Maxillofac Surg 2009; 67: 1806-1814.

11. Leung $Y Y$, Cheung L K. Safety of coronectomy versus excision of wisdom teeth: a randomized controlled trial. Oral Surg Oral Med Oral Pathol Oral Radiol Endod 2009; 108: 821-827.

12. Dolanmaz D, Yildirim G, Isik K, Kucuk K, Ozturk A. A preferable technique for protecting the inferior alveolar nerve: coronectomy. J Oral Maxillofac Surg 2009; 67: 1234-1238.

13. Pogrel M A. An update on coronectomy. J Oral Maxillofac Surg 2009; 67: 1782-1783.

14. Drage N A, Renton T. Inferior alveolar nerve injury related to mandibular third molar surgery: an unusual case presentation. Oral Surg Oral Med Oral Pathol Oral Radiol Endod 2002; 93: 358-361.

15. Umar G, Bryant C, Obisesan O, Rood J P. Correlation of the radiological predictive factors of inferior alveolar nerve injury with cone beam computed tomography findings. J Oral Surg 2010; 3: 72-82. 